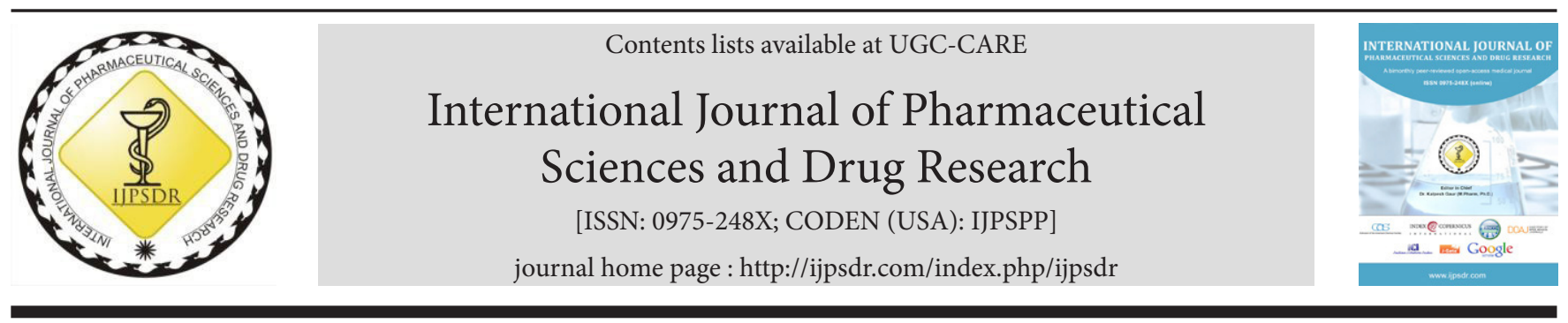

Research Article

\title{
In vitro Antioxidant Potentiality of Purified Anthocyanin from Floral Petals of Wild Balsam Species
}

\author{
Rajendranpillai Arathy ${ }^{1}$, Kumaraswamy Murugan ${ }^{2 *}$, Kalekkal Vasupillai Dinesh Babu ${ }^{3}$, Gopal Sarayu Manoj ${ }^{1}$ \\ ${ }^{1}$ Department of Botany, Mahatma Gandhi College, Thiruvananthapuram-695004, Kerala, India \\ ${ }^{2}$ Center for Innovation in Science and Social Action (CISSA), Thiruvananthapuram-695010, Kerala, India \\ ${ }^{3}$ Department of Chemistry, Government College for Women, Thiruvananthapuram-695014, Kerala, India
}

\begin{tabular}{l} 
A R T I C L E I N F O \\
\hline Article history: \\
Received: 26 March, 2020 \\
Revised: 09 June, 2020 \\
Accepted: 16 June, 2020 \\
Published: 30 July, 2020 \\
Keywords: \\
Anthocyanin, \\
Antioxidants, \\
Free radical scavenging, \\
Purification, \\
Wild balsam. \\
D0I: \\
10.25004/IJPSDR.2020.120402
\end{tabular}

\section{INTRODUCTION}

Antioxidants (AOX) can mitigate oxidative damage to food products during processing, storage, and preparation of food. AOXs may help in designing more healthy food with reduced levels of lipid and protein oxidation products. Vegetables and fruits or traditional herbal drugs from time immemorial are used as food or for the ailment of headache, hemoptysis, asthma, obesity, constipation, hemorrhoids, and goat. It is also noticed as an aphrodisiac, emmenagogue, and diuretic agent in folk drugs. ${ }^{[1,2]}$ There is only limited information is available about the AOX

\begin{abstract}
A B S T R A C T
Phenolics are the largest group of phytochemicals ubiquitous in plant species with considerable interest economically. Recently, search of novel polyphenols increasingly becomes an area of intensive pharmacological research due to their multiple bioactive features, such as, antioxidant (AOX), antimicrobial, anti-carcinogenic, anti-viral, and anti-inflammatory potentialities. Anthocyanins are flavonoid groups of polyphenols, a group predominant in flowers, fruits, and vegetables. The flavonoids, perhaps the unique single group of phenolics in foods, comprise a group of over $4,200 \mathrm{C}_{15}$ aromatic molecules with multiple structural patterns. The functions of anthocyanins as medicinal have been well-accepted in folk medicine throughout the world. In fact, these molecules are connected to an amazingly broad-based range of health benefits. At this juncture, the aim of this work was to evaluate the AOX activities of purified anthocyanin from wild balsam species. Initially, anthocyanin was extracted from floral leaves of wild balsam species and purified by chromatographic techniques. Subsequently, it was subjected to nuclear magnetic resonance (NMR) and liquid chromatography coupled to mass spectrometry (LC MS) analysis. The major fractions identified were hesperidin, dimethoxy antirrhinin, and trimethoxy antirrhinin. Further, the anthocyanin extracts were subjected to in vitro protocols like 2,2'-azino-bis-3-ethylbenzothiazoline-6-sulphonic acid (ABTS) radical cation, 2,2-diphenyl-1-picrylhydrazyl (DPPH) scavenging assay, ferric reducing AOX power (FRAP), beta carotene bleaching assay, metal chelating, and $\mathrm{H}_{2} \mathrm{O}_{2}$ scavenging power. Interestingly, ABTS, FRAP analyzes yielded significant results, as compared to others. The data were comparable with that of synthetic AOXs like ascorbate and catechin. Meanwhile, beta carotene and $\mathrm{H}_{2} \mathrm{O}_{2}$ scavenging assay showed moderate results. DPPH and metal chelating protocols displayed the values 70.98 and $64.66 \%$, respectively, at $25 \mu \mathrm{g} / \mathrm{mL}$ concentration. This study provides model systems for the evaluation of natural AOXs, like anthocyanin. Future in vivo clinical studies are warranted to confirm the obtained data.
\end{abstract}

\footnotetext{
${ }^{*}$ Corresponding Author: Kumaraswamy Murugan

Address: Center for Innovation in Science and Social Action (CISSA), Thiruvananthapuram-695010, Kerala, India

Email $\square$ : harimurukan@gmail.com

Relevant conflicts of interest/financial disclosures: The authors declare that the research was conducted in the absence of any commercial or financial relationships that could be construed as a potential conflict of interest.

Copyright (C) 2020 Rajendranpillai Arathy et al. This is an open access article distributed under the terms of the Creative Commons AttributionNonCommercial-ShareAlike 4.0 International License which allows others to remix, tweak, and build upon the work non-commercially, as long as the author is credited and the new creations are licensed under the identical terms.
}

activities of different polarity extracts of plants grown widely under different climatic conditions. Synthetic AOXs were used enormously in food industries to prevent oxidative reactions in living organisms. However, because of potential health hazards associated with dietary intake of these chemical AOXs, the focus of biologists is to explore plant-based compounds as natural AOXs.

The AOX research an area in the pharmaceutical field as well as in the food industry. Herbals, particularly medicinal ones, have been largely studied for their AOX power for the past several years. AOXs phytochemicals 
from aromatic, spicy, medicinal, and other herbals were screened to isolate natural AOX formulations for food, cosmetic, and other applications. ${ }^{[3]}$ It is also documented that higher intake of natural AOXs rich food is connected with lower risks of degenerative disorders, particularly lifestyle disorders. Anthocyanins are accepted as the largest group of water-soluble pigments in nature with diversified colors in petals like blue, purple, red, and orange. Anthocyanin refers to anthos mean flowers, and kyanos, dark blue. ${ }^{[4]}$ Common sources of anthocyanins were blueberries, cherries, raspberries, strawberries, black currants, purple grapes, and red wine. Anthocyanin belongs to flavonoids, but they are distinguished due to their capacity to form flavylium cations. In the present study, AOX potentiality of purified anthocyanin from wild balsam species is analyzed by different in vitro protocols.

\section{Materials AND METHODS}

\section{Plant Materials}

For the whole study, the fresh, healthy flowers of Impatiens balsamina (wild) collected from the natural habitats of hilly regions of Trivandrum, Kerala.

\section{Estimation of Anthocyanin Content}

1-gram flower was homogenized with acidified methanol $(1 \% \mathrm{HCl})$, and the anthocyanin content was quantified by the standard protocol of Sutharut and Sudarat. ${ }^{[5]}$ The absorbance of each dilution was read at 510 and $700 \mathrm{~nm}$ against distilled water as blank.

\section{Purification and Characterization of Anthocyanin from Wild Balsam}

Anthocyanin from fresh flowers of wild balsam was extracted by acidified methanol $(100 \%$ methanol: $1 \% \mathrm{HCl}$, 99:1, v/v), i.e., the petals were immersed in acidified methanol for overnight in dark at low temperature $\left(4^{\circ} \mathrm{C}\right)$. Subsequently, the crude solution obtained was filtered through cheesecloth and concentrated in rotary vacuum evaporator under vacuum at temperature not exceeding $40^{\circ} \mathrm{C}$. Then, the aqueous extract was partitioned with ethyl acetate, and the remaining water extract was concentrated in rotary vacuum evaporator, temperature not exceeding $40^{\circ} \mathrm{C}$. It was further fractionated by using Sephadex LH-20, followed by C18 as separation matrices. The aqueous extract was loaded on to a Sephadex LH-20 using $100 \%$ methanol. Based on the TLC profile, the fractions were subjected to purification by C-18 open-column chromatography using acidified water $(1 \% \mathrm{HCl})$, and the column was eluted with a gradient of acidified methanol $(1 \% \mathrm{HCl})$ and water $(0: 100$ to $50: 50)$. From reverse phased (RP) C-18 open-column chromatography, based on the TLC profile, the pure fractions were concentrated and kept at low temperature $\left(-20^{\circ} \mathrm{C}\right)$. Purified anthocyanin from the wild balsam was subjected to NMR analysis for their structural identification.

\section{AOX Activity}

\section{Hydrogen Peroxide Scavenging}

Hydrogen peroxide scavenging activity was determined according to a previously described method of Ruch et al. ${ }^{[6]}$

\section{DPPH Assay}

AOX activity was also determined by DPPH assay using the standard protocol of Saeed et al. ${ }^{[7]}$

\section{Metal Chelating Activity}

The chelation of ferrous ions was estimated by the method of Dinis et al. ${ }^{[8]}$ The absorbance of the solution was, thereafter, measured at $562 \mathrm{~nm}$.

\section{ABTS Radical Scavenging Activity}

ABTS (2,2'-azino-bis(3-ethylbenzothiazoline-6-sulphonic) acid) assay was performed according to the protocol of Delgado-Andrade et al. ${ }^{[9]}$

\section{FRAP Assay}

The FRAP assay was carried as per the proven protocol of Benzie and Strain. ${ }^{[10]}$ The principle of this method was based on the reduction of ferric-tripyridyl triazine complex to ferrous colored form in the presence of AOXs.

\section{$\beta$-Carotene Bleaching (BCB) Test}

AOX activity of anthocyanin was determined according to a slightly modified version of the $\beta$-carotene bleaching (BCB) method. $^{[11]}$

\section{Statistical Analysis}

SPSS 18.0 statistical software was used for data analysis. Data from different assay treatments were analyzed by one-way analysis of variance (ANOVA). The Student-Neuman-Keuls post-hoc test was used to determine significance for individual experimental conditions. Differences with $\mathrm{p}<0.01$ or $\mathrm{p}<0.05$ were regarded as significant.

\section{RESULTS}

\section{Anthocyanin Content, Purification, and Fractionation}

The total anthocyanin content in the floral leaves of wild balsam was $5.88 \mathrm{mg} / \mathrm{g}$. Purified anthocyanins after liquid-liquid partition protocol, Sephadex LH-20 gel chromatography, and RP C18 open column chromatography were subjected to NMR and LC MS analysis (Figs 1 to 3) for their structural identification and were identified as hesperidin, dimethoxy antirrhinin, and trimethoxy antirrhinin (Figs $4 \mathrm{~A}$ to $\mathrm{C}$ ).

\section{AOX Activity}

AOX potentialities of the cultivars were evaluated using different assays. 

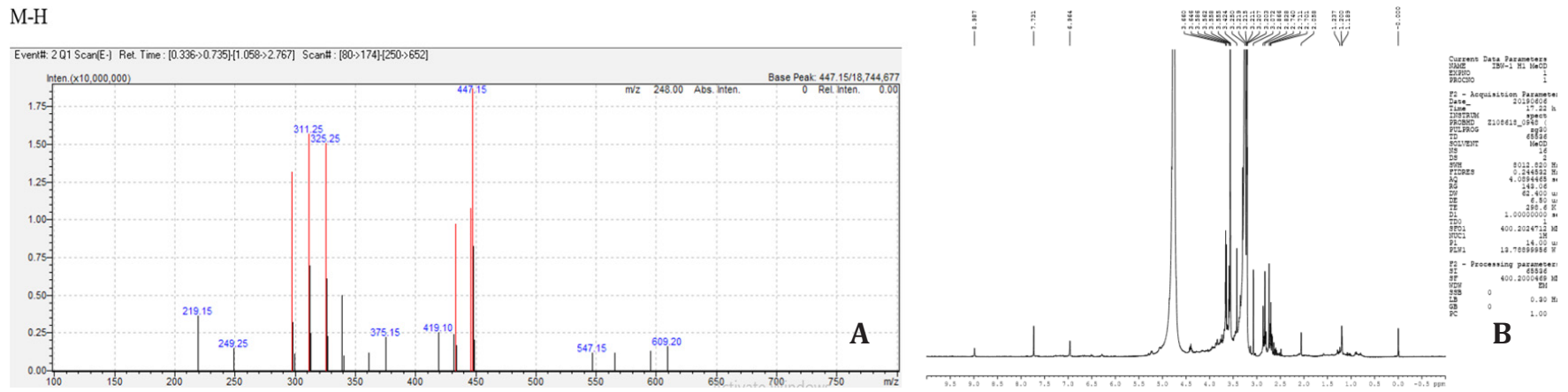

Fig. 1: (a) LC MS, and (b) NMR spectrum of hesperidin from the wild balsam

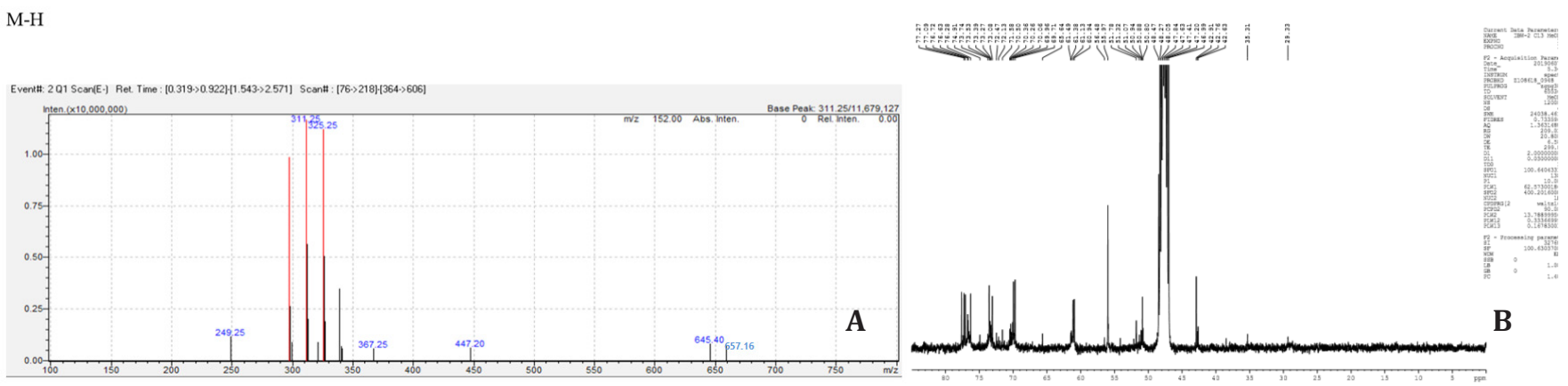

Fig. 2: (a) LC MS, and (b) NMR spectrum of dimethoxy antirrhinin from the wild balsam

M-H

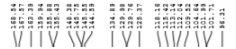
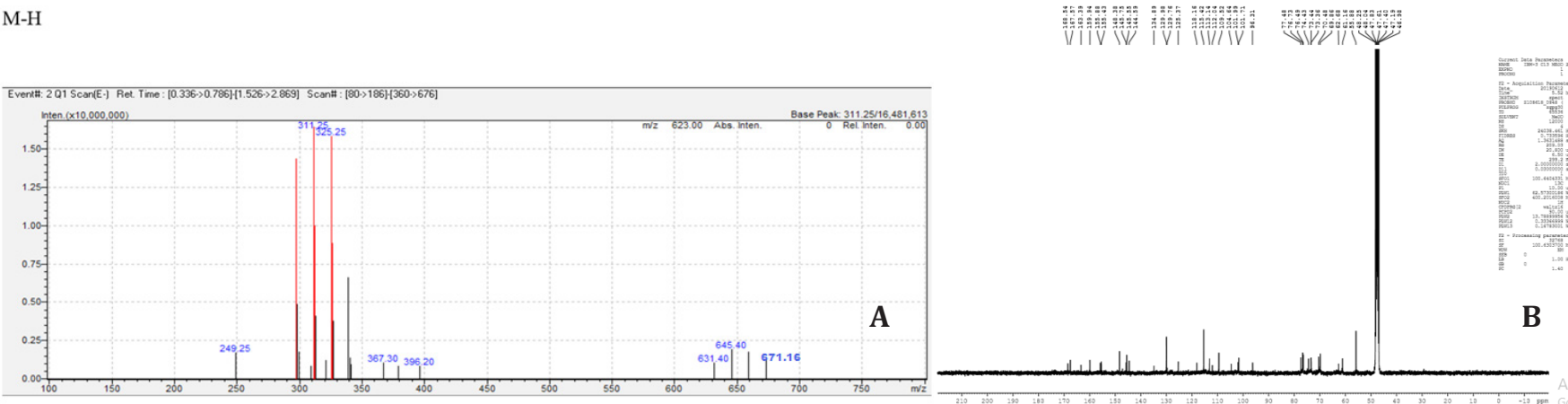

Fig. 3: (a) LC MS, and (b) NMR spectrum of trimethoxy antirrhinin from the wild balsam<smiles>COc1ccc([C@H]2CC(=O)c3c(O)cc(OC4O[C@H](CO[C@H]5OC(C)[C@H](O)[C@H](O)[C@H]5O)[C@@H](O)[C@H](O)[C@H]4O)cc3O2)cc1O</smiles><smiles>COc1cc(OC)c2cc(OC3O[C@H](COC4O[C@H](C)[C@@H](O)[C@H](O)[C@H]4O)[C@@H](O)[C@H](O)[C@H]3O)c(-c3ccc(O)c(O)c3)cc2c1</smiles><smiles>COc1cc(OC)c2cc(OC3O[C@H](COC4O[C@H](C)[C@@H](O)[C@H](O)[C@H]4O)[C@@H](O)[C@H](O)[C@H]3O)c(-c3ccc(OC)c(O)c3)cc2c1</smiles>

Fig. 4: Chemical structure of (A) Hesperidin $\left(\mathrm{C}_{28} \mathrm{H}_{34} \mathrm{O}_{15}\right)$; (B) Dimethoxy antirrhinin $\left(\mathrm{C}_{29} \mathrm{H}_{35} \mathrm{ClO}_{15}\right)$;

(C) Trimethoxy antirrhinin $\left(\mathrm{C}_{30} \mathrm{H}_{37} \mathrm{ClO}_{15}\right)$

Hydrogen Peroxide $\left(\mathrm{H}_{2} \mathrm{O}_{2}\right)$ Radical Scavenging

The purified anthocyanin activity from wild balsam species has been determined by following the rate of $\mathrm{H}_{2} \mathrm{O}_{2}$ consumption in an incubation system $\left(\mathrm{H}_{2} \mathrm{O}_{2}+\right.$ scavenger $)$ using the UV method. The result of the $\mathrm{H}_{2} \mathrm{O}_{2}$ scavenging test of the purified anthocyanin extract in comparison with the standard (ascorbic acid and catechin) at $230 \mathrm{~nm}$ was shown in Table 1. The percentage of inhibition against different 
concentrations, as well as, synthetic drug was used to plot the standard curve which was used to calculate the $\mathrm{IC}_{50}(\mu \mathrm{g} / \mathrm{mL})$ of the sample which was determined as 18.5 , 16 , and $13.5 \mu \mathrm{g} / \mathrm{mL}$ for anthocyanin extract, catechin, and ascorbate, respectively.

\section{DPPH Radical Scavenging Assay}

DPPH has been generally used to analyze the free radical scavenging effectiveness of AOX molecules. In the DPPH assay, the AOXs were able to reduce the stable radical DPPH to the yellow-colored diphenyl-picryl hydrazine at $517 \mathrm{~nm}$. The results are expressed as the $\mathrm{IC}_{50}$ value (the amount of AOX necessary to decrease the initial DPPH concentration by $50 \%$ ). The results of the DPPH radical scavenging test of purified anthocyanin extract in comparison with the standard (catechin and ascorbic acid) were shown in Table 2. The percentage of inhibition against different concentrations of extracts of the balsam, as well as, ascorbate, catechin were used to plot the standard curve which was used to calculate the $\mathrm{IC}_{50}(\mu \mathrm{g} / \mathrm{mL})$ of each sample, which was determined as $16.25 \mu \mathrm{g} / \mathrm{mL}$ in anthocyanin extract, $10 \mu \mathrm{g} / \mathrm{mL}$ in catechin, and $12.07 \mu \mathrm{g} / \mathrm{mL}$ in ascorbate, respectively.

Table 1: Hydrogen peroxide scavenging activities from the petals of wild balsam

\begin{tabular}{ll}
\hline Concentration $(\mu \mathrm{g} / \mathrm{mL})$ & \% inhibition \\
\hline 5 & \\
10 & 28.578 \\
15 & 32.87 \\
20 & 43.11 \\
25 & 54.23 \\
Catechin $(25 \mu \mathrm{g} / \mathrm{mL})$ & 55.11 \\
Ascorbate $(25 \mu \mathrm{g} / \mathrm{mL})$ & 77.9 \\
F ratio & 77.5 \\
SE & $20,967^{* *}$ \\
CD & 0.0278 \\
\hline
\end{tabular}

\section{$* *$ indicates $\mathrm{p}<0.01$}

Table 2: DPPH radical-scavenging activities from the petals of wild balsam

\begin{tabular}{ll}
\hline Concentration $(\mu \mathrm{g} / \mathrm{mL})$ & \% inhibition \\
\hline 5 & 16.44 \\
10 & 32.32 \\
15 & 39.18 \\
20 & 61.86 \\
25 & 70.98 \\
Catechin $(25 \mu \mathrm{g} / \mathrm{mL})$ & 86 \\
Ascorbate $(25 \mu \mathrm{g} / \mathrm{mL})$ & 84 \\
F ratio & $34,512^{* *}$ \\
SE & 0.0541 \\
CD & 0.0043 \\
** indicates $\mathrm{p}<0.01$ &
\end{tabular}

** indicates $\mathrm{p}<0.01$

\section{ABTS Radical Scavenging Assay}

In the ABTS assay, the radical is converted into its radical cation by addition of sodium persulfate. This radical cation is blue colored and absorbs light at $734 \mathrm{~nm}$. The ABTS radical cation is reactive towards the AOXs, including phenolics, thiols, and vitamin C. On the onset of the reaction, the blue ABTS radical cation is transformed back to its colorless neutral form. The reaction may be monitored spectrophotometrically. This assay is known as the Trolox equivalent AOX capacity (TEAC) assay. The results of the ABTS radical scavenging test of the anthocyanin extracts in comparison with the standard (Trolox) were shown in Table 3. The percentage of inhibition against different concentrations of anthocyanin extracts in comparison with the standard, as well as, Trolox as standard, was used to plot the standard curve. This curve was used to calculate the $\mathrm{IC}_{50}(\mu \mathrm{g} / \mathrm{mL})$ of each sample, which was determined as $8.05 \mu \mathrm{g} / \mathrm{mL}$ in anthocyanin and $5.89 \mu \mathrm{g} / \mathrm{mL}$ in Trolox, respectively. It is also observed that percentage inhibition values go on increasing with increases in dosage of plant extracts in the assay mixture.

\section{FRAP Assay}

In this assay, the yellow color of the test solution changes to different green and blue shades depending upon the reducing potential of the AOX molecule. The presence of radicals (i.e., AOXs) facilitates the conversion of the $\mathrm{Fe}^{3+} /$ ferricyanide complex employed in this protocol to the ferrous form indicated by the synthesis of pearls Prussian blue at $700 \mathrm{~nm}$. The data of the FRAP test of the purified anthocyanin extract in comparison with the standard (catechin and ascorbate) at $700 \mathrm{~nm}$ is shown in Table 4. The percentage of inhibition against different concentrations of anthocyanin extracts, as well as, catechin and ascorbate was used to plot the standard curve which was used to calculate the $\mathrm{IC}_{50}(\mu \mathrm{g} / \mathrm{mL})$ of each sample, which was determined as 4.04, 4, and $3.29 \mu \mathrm{g} / \mathrm{mL}$ for anthocyanin extract, catechin, and ascorbic acid, respectively. It is also observed that inhibition percentage values go on increasing with an increase in the dosage of anthocyanin extracts in the assay mixture.

Table 3: ABTS radical-scavenging activities in petals of wild balsam

\begin{tabular}{ll}
\hline Concentration $(\mu \mathrm{g} / \mathrm{mL})$ & $\%$ inhibition \\
\hline 5 & 35.08 \\
10 & 56.03 \\
15 & 69.43 \\
20 & 80.13 \\
25 & 85.9 \\
Trolox $(25 \mu \mathrm{g} / \mathrm{mL})$ & 88.4 \\
F ratio & $1,845^{* *}$ \\
SE & 0.06712 \\
CD & 0.003 \\
\hline
\end{tabular}

** indicates $\mathrm{p}<0.01$ 
In vitro Antioxidant Potentiality of Purified Anthocyanin from Balsam Floral Petals

Table 4: FRAP assay activities in the petals of wild balsam

\begin{tabular}{ll}
\hline Concentration $(\mu \mathrm{g} / \mathrm{mL})$ & \% inhibition \\
\hline 5 & 52.52 \\
10 & 65.08 \\
15 & 68.66 \\
20 & 79.5 \\
25 & 81.89 \\
Catechin $(25 \mu \mathrm{g} / \mathrm{mL})$ & 86.4 \\
Ascorbate $(25 \mu \mathrm{g} / \mathrm{mL})$ & 93 \\
F ratio & $19,432^{* *}$ \\
SE & 0.0341 \\
CD & 0.0091 \\
\hline
\end{tabular}

** indicates $\mathrm{p}<0.01$

Table 5: Metal chelating activities in the petals of wild balsam

\begin{tabular}{ll}
\hline Concentration $(\mu \mathrm{g} / \mathrm{mL})$ & \% inhibition \\
\hline 5 & 31.59 \\
10 & 44.97 \\
15 & 56.49 \\
20 & 61.84 \\
25 & 64.66 \\
Catechin $(25 \mu \mathrm{g} / \mathrm{mL})$ & 98 \\
Ascorbate $(25 \mu \mathrm{g} / \mathrm{mL})$ & 97 \\
F ratio & $14,532^{* *}$ \\
SE & 0.0054 \\
CD & 0.0017 \\
\hline
\end{tabular}

$* *$ indicates $\mathrm{p}<0.01$

\section{Metal Chelating Activity}

Free iron generation results in induction and formation of reactive oxygen species (ROSs)/ free radicals in biological systems. Secondary metabolites of herbal extracts are empowered with metal chelating potentiality. In the present study, the anthocyanin concentration ranged from 5 to $25 \mu \mathrm{g} / \mathrm{mL}$ was tested for the same. Interestingly, the metal chelating activity also displayed an increase with concentrations of anthocyanin content. The percentage of inhibition of metal-chelating ranged from 31.59 to $64.66 \%$. The $\%$ of inhibition of free radicals by various doses of anthocyanin content was more or less at par with that of the respective standards like catechin and ascorbate (Table 5).

\section{$\beta$-Carotene Linoleic Acid Assay}

Synthetic ABTS and DPPH scavenging models are ideal protocols to indicate the AOX ability of herbal extracts; however, not reliable in food or biologically relevant oxidizable substrate protective systems. ${ }^{[12]}$ So, the $\beta$-carotene/linoleic acid (lipid-water emulsion) assay was used to analyze the potentiality of anthocyanin. In this method, linoleic acid oxidation produces hydroperoxide-derived free radicals that attack the chromophore of $\beta$-carotene, resulting in bleaching of the emulsion product. Compounds capable
Table 6: $\beta$-carotene bleaching activities in the petals of wild balsam

\begin{tabular}{ll}
\hline Concentration $(\mu \mathrm{g} / \mathrm{mL})$ & \% inhibition \\
\hline 5 & 22.32 \\
10 & 28.54 \\
15 & 36.09 \\
20 & 48.21 \\
25 & 51.08 \\
Catechin $(25 \mu \mathrm{g} / \mathrm{mL})$ & 77.9 \\
Ascorbate $(25 \mu \mathrm{g} / \mathrm{mL})$ & 77.5 \\
F ratio & $23,145.6^{* *}$ \\
SE & 0.0087 \\
CD & 0.021 \\
\hline
\end{tabular}

** indicates $\mathrm{p}<0.01$

of limiting/ inhibiting the oxidation of $\beta$-carotene may be considered as free radical scavenger and primary AOX, as shown in the Table 6 that anthocyanin of balsam species was capable of inhibiting the bleaching of $\beta$-carotene by scavenging linoleate-derived free radicals. $\beta$-carotene linoleic acid activity of wild balsam anthocyanin at $25 \mu \mathrm{g} / \mathrm{mL}$ concentration was found to be $51.08 \%$ (Table 6).

\section{Discussion}

Globally, the herbal species are trailed widely as a source of phytotherapeutic drugs, especially in the area of AOX potentiality. ${ }^{[13]}$ In plants, the polyphenolics form the major AOXs due to their aromatic ring that allows the stabilization and relocation of the unpaired electrons via donating hydrogen and electrons from their hydroxyl groups. Generally, the secondary metabolite content shows diversity among the plant species. Similarly, the content varies in the tissues and developmental part of their life cycle. Environmental factors, such as, temperature, water, and light regimes also regulate its synthesis. ${ }^{[14]}$

Tropical plant species are adapted morphologically and physiologically to survive in habitats with extreme environmental conditions, like high temperatures, water scarcity, and light irradiation. One of the adaptations is the mitigation of oxidative stress via balancing ROS under extreme levels and also, efficient signaling mechanism. Molecular crosstalk, epigenetic memories, ROS signaling, accumulation of plant hormones, such as, salicylic acid, ethylene, jasmonic acid, and abscisic acid, change in redox status and inorganic ion fluxes, R-gene resistance, and systemic acquired resistance (SAR) are some of the modifications/ mechanisms adopted by plants to adapt and defend themselves from the environmental stress. ${ }^{[15]}$ Plant phytochemicals, specifically phenolics, can adjust the ROS level, thus activating a network of biochemical reactions to improve tolerance. Therefore, the AOX activities of plant species are important to survive under these extreme environmental conditions.

The anthocyanin content of balsam species was $5.88 \mathrm{mg} / \mathrm{g}$. Purified anthocyanins possess hesperidin, 
dimethoxy antirrhinin, and trimethoxy antirrhinin molecules. These molecules form major groups of primary AOXs or free radical terminators. Studies in rose (Rosa hybrida) varieties reflect that the varieties with dark colors were richest in anthocyanin content than the light-colored varieties. ${ }^{[16]}$ A similar trend was also observed for AOX activity, i.e., the dark color can serve as an indicator of AOX activity.

The AOX potentiality of purified anthocyanins extracts can be quantified by different protocols, and the results of the test may be used to establish AOX ranking. In the present study, the six methods were used to evaluate the AOX activity. Furthermore, all the protocols presented significant values statistically. Many studies have documented this correlation, and some researchers proposed high correlation between the DPPH and FRAP methods as marker of AOX power of molecules. ${ }^{[17]}$ Due to the presence of multiple radicals, and the changes in the nature of AOXs, there is no single and universal method to characterize the AOX activities of all molecules. ${ }^{[18]}$ These reports conclude that any of the methods used in the present study could be used to determine and categorize the AOX activity of species.

Comparison of the six methods in which ascorbate and catchin were used as reference molecules, it can be noted that the evaluated AOX activity was not the same. The values obtained by ABTS and FRAP were more or less similar, whereas those obtained by $\mathrm{H}_{2} \mathrm{O}_{2}$ and $\beta$-carotene were lower; in fact, these differences depended on the mode of action of the anthocyanin. Specifically, AOX activity of ABTS was highest than FRAP, depending on the anthocyanin specificity of activity (Table 3). Studies in woody species of semiarid zones of Mexico, exhibited that the AOX activity by DPPH was superior than ABTS, i.e., the values of both methods differed among the species. ${ }^{[19]}$ In fact, in Eucaliptus camaldulensis provided similar values with the methods. Likewise, in studies conducted with medicinal plants, different values were documented for the AOX activity quantified by ABTS and DPPH, although, in this case, the optimal values were noticed by ABTS. ${ }^{[20]}$ Thus, it is important to note that AOX molecules can respond in a different manner to various radical or oxidant molecules. Studies also showed that the higher or lower activity attributed to a species depended on the protocols employed, such as, ABTS, DPPH, and FRAP, although, in disagreement with the present results. Interestingly, it was reported that Pistacia lentiscus showed the highest AOX activity by DPPH and reductive power assay ${ }^{[21]}$ than by other protocols. The present study highlights that categorization of drugs based on AOX protocols may serve as a useful tool for the selection of species with higher or lower AOX activities.

Thus, the present work may be concluded that hesperidin, dimethoxy antirrhinin, and trimethoxy antirrhinin were the major purified anthocyanin obtained from wild balsam species. The AOX results obtained in this study via the six methods suggest the potentiality of balsam anthocyanin as efficient AOX. The protocols also vary in sensitivity when establishing variations in the AOX activity of the species. Thus, with some of the protocols, the variation is lower than that obtained with other methods. Future studies are warranted to confirm the obtained data by in vivo animal models.

\section{ACKNOWLEDGEMENT}

This study was supported by the ASPIRE fellowship of the Department of Collegiate Education, Government of Kerala.

\section{REFERENCES}

1. Sadeghi M, Zolfaghari B, Senatore M, Lanzotti V. Spirostane, Furostane and Cholestane Saponins from Persian leek with Antifungal Activity. Food Chem. 2013;141(2):1512-1521.

2. Feghhi-Najafabadi S, Safaeian L, Zolfaghari B. In vitro Antioxidant Effects of Different Extracts Obtained from the Leaves and Seeds of Allium Ampeloprasum Subsp. Persicum. J Herbmed Pharmacol. 2019;9(3):1-5.

3. ElkhamLichia A, Hajajia HEl, Farajb H, Alamib A, Balia BEl, Lachkar M. Phytochemical Screening and Evaluation of Antioxidant and Antibacterial Activities of Seeds and Pods Extracts of Calycotome Villosa Subsp. Intermedia Journal of Applied Pharmaceutical Science. 2017;7(04):192-198.

4. Miguel MG. Anthocyanins: Antioxidant and/or Anti-inflammatory Activities. Journal of Applied Pharmaceutical Science. 2011;1(6): 7-15.

5. Sutharut J, Sudarat J. Total Anthocyanin Content and Antioxidant Activity of Germinated Colored Rice. Inter Food Res. J. 2012; 19:215-221.

6. Ruch RJ, Cheng SJ, Klaunig JE. Prevention of Cytotoxicity and inhibition of intracellular communication by antioxidant catechins isolated from chinese green tea. Carcinogenesis. 1989;10:1003-1008.

7. Saeed N, Khan MR, Shabbir M. Antioxidant activity, total phenolic and total flavonoid contents of whole plant extracts Torilis leptophylla L. BMC Complement Altern Med. 2012;12:1-12.

8. Dinis TCP, Madeira VMC, Almeida LM. Action of phenolic derivatives (acetaminophen, salicylate and 5-aminosalicylate) as inhibitors of membrane lipid peroxidation and as peroxyl radical scavengers. Arch Biochem Biophys. 1994;315(1):161-169.

9. Delgado-Andrade C, Rufian-Henares JA, Morales FJ. Assessing the antioxidant activity of melanoidins from coffee brews by different antioxidant methods. J Agric Food Chem. 2005;53:7832-7840.

10. Benzie IFF, Strain JJ. Ferric Reducing/Antioxidant Power Assay: Direct Measure of Total Antioxidant Activity of Biological Fluids and Modified Version for Simultaneous Measurement of Total Antioxidant Power and Ascorbic Acid Concentration. Meth in Enz. 1999;299:15-27.

11. Pratt DE. Natural Antioxidants of Soybean and other Oil-Seeds. Autoxidation in Food and Biological Systems. M. G. Simic, and M. Karel (Eds.), New York: Plenum Press, 1980.

12. Abo El-Maati MF, Labib SM, Al-Gaby AMA, Ramadan MF. Antioxidant Properties of Different Extracts from Five Medicinal Plants. Zagazig J Agric Res. 2012;39(4).

13. Ayoub Z, Mehta A, Mishra SK, Ahirwal L. Medicinal Plants as Natural Antioxidants: A Review. Journal of Botanical Society. 2017;48:ISSN 2229-7170.

14. Ramakrishna A, Ravishankar GA. Influence of Abiotic Stress Signals on Secondary Metabolites in Plants. Plant Signal Behav. 2011;6(11):1720-1731.

15. Devi EL, Kumar S, Basanta Singh T, Sharma SK, Beemrote A, Devi CP, et al. Adaptation Strategies and Defence Mechanisms of Plants 
During Environmental Stress. Medicinal Plants and Environmental Challenges. 2017;359-413.

16. Poonam Kumari, Raju DVS, Prasad KV, Singh KP, Saha S, Arora A, et al. Quantification and Correlation of Anthocyanin Pigments and their Antioxidant Activities in Rose (Rosa Hybrida) Varieties. Indian Journal of Agricultural Sciences. 2017;87(10):1340-1346.

17. Shah P, Modi HA. Comparative Study of DPPH, ABTS and FRAP Assays for Determination of Antioxidant Activity. International Journal for Research in Applied Science and Engineering Technology. 2015;3(4):ISSN: 2321-9653.

18. Lewoyehu M, Amare M . Comparative Evaluation of Analytical Methods for Determining the Antioxidant Activities of Honey: A
Review. Cogent Food and Agriculture. 2019;5:2331-1932.

19. Wong-Paz JE, Contreras-Esquivel JC, Rodriguez-Herrera R, Carrillo-Inungaray ML, Lopez LI, Nevarez-Moorillon GV, Aguilar CN. Total Phenolic Content, In vitro Antioxidant Activity and Chemical Composition of Plant Extracts from Semiarid Mexican Region. Asian Pac J Trop Med. 2015;8:104-111.

20. Rajurkar NS, Hande SM. Estimation of Phytochemical Content and Antioxidant Activity of Some Selected Traditional Indian Medicinal Plants. Indian J Pharm Sci. 2011;73:146-151.

21. Gonzalez-Burgos E, Gomez-Serranillos MP. Terpene Compounds in Nature: A Review of Their Potential Antioxidant Activity. Curr Med Chem. 2012;19:5319-5341.

HOW TO CITE THIS ARTICLE: Arathy R, Murugan K, Babu KVD, Manoj GS. In vitro antioxidant potentiality of purified anthocyanin from floral petals of wild balsam species. Int. J. Pharm. Sci. Drug Res. 2020;12(4):313-319. DOI: 10.25004/IJPSDR.2020.120402 